\title{
Mesenchymal stem cell-based therapy in kidney transplantation
}

\author{
Cheng Chen and Jianquan Hou
}

\begin{abstract}
Kidney transplantation is the best treatment for end-stage renal disease, but its implementation is limited by organ shortage and immune rejection. Side effects of current immunosuppressive drugs, such as nephrotoxicity, opportunistic infection, and tumorigenic potential, influence long-term graft outcomes. In recent years, continued research and subsequent discoveries concerning the properties and potential utilization of mesenchymal stem cells (MSCs) have aroused considerable interest and expectations. Biological characteristics of MSCs, including multi-lineage differentiation, homing potential, paracrine effect and immunomodulation, have opened new horizons for applications in kidney transplantation. However, many studies have shown that the biological activity of MSCs depends on internal inflammatory conditions, and the safety and efficacy of the clinical application of MSCs remain controversial. This review summarizes the findings of a large number of studies and aims to provide an objective viewpoint based on a comprehensive analysis of the presently established benefits and obstacles of implementing MSC-based therapy in kidney transplantation, and to promote its clinical translation.
\end{abstract}

\section{Background}

The number of patients with chronic kidney disease and end-stage renal disease (ESRD) increases each year. Kidney transplantation is an effective long-term means of treatment for ESRD, but is closely accompanied by a high risk of post-transplant rejection. With improvements in tissue type matching and the use of new immunosuppressive agents, the danger of acute renal post-transplant rejection has been greatly reduced, but the hazards for long-term graft survival have remained unchanged.

Regular immunosuppressive drug protocols mainly include corticosteroids in combination with calcineurin inhibitors, purine/pyrimidine synthesis inhibitors, and sometimes lymphodepletion [1]. However, their use should be gradually tapered off to lower the risk of opportunistic infections and malignancies, as well as to prevent long-term toxicity to the central nervous, gastrointestinal and hematopoietic systems and kidneys. Therefore, there is a substantial need for novel immunosuppressive therapies that have few side effects, while maintaining efficacy.

Mesenchymal stem cells (MSCs) are mesodermderived multipotent stromal cells that have high self-

\footnotetext{
* Correspondence: houjianquan@suda.edu.cn

Department of Urology, The First Affiliated Hospital of Soochow University, 188 Shizi Road, Suzhou 215006, Jiangsu, PR China
}

renewal and multi-lineage differentiation potential, an immunomodulatory and inflammatory homing capacity, and the ability to repair damaged tissues and organs. They are widespread in many tissues of the body and can be cultured and multiplied in vitro, with the ability to differentiate into bone, cartilage, fat, muscle, nerves and other tissues under certain conditions [2,3].

The underlying immunomodulatory mechanism of action of MSCs has been widely recognized, and its effectiveness has also been confirmed by early clinical trials in the treatment of graft-versus-host disease (GVHD) [4]. Thus, we can speculate that the application of MSCs in kidney transplantation will not only reduce the amount of immunosuppressive drugs required, but also enhance the renal function of patients and improve their longterm survival.

\section{Biological characteristics of MSCs}

MSCs are pluripotent, long, shuttle-shaped, fibroblastlike stem cells that are present in almost all tissues and organs, including the bone marrow, periosteum, fat tissue, pulp, synovium, umbilical cord, placenta, amniotic fluid and fetal tissues.

To date, no studies have reported the discovery of specific MSC surface markers. In view of this, in 2006, the International Society of Cellular Therapy defined MSCs 
by the following three minimum criteria [5]: (1) MSCs must adhere to plastic under standard tissue culture conditions; (2) MSCs must express specific cell surface markers such as CD73, CD90 and CD105, while lacking the expression of hematopoietic stem cell markers and autologous transplant rejection antigens such as CD45, CD34, CD14, CD11b, CD79 $\alpha$, CD19 and human leukocyte antigen (HLA)-DR; (3) in vitro, these cells must have the capacity to be induced to differentiate into adipocytes, osteoblasts and chondrocytes.

MSCs have multi-lineage differentiation potential; in vitro, they can differentiate into mesoderm lineage (bone, cartilage, fat, and connective tissues) and transdifferentiate into both ectodermal (nerve and epithelial tissues) and endodermal cells (muscle and liver cells) [2, 3]. Thus, MSCs have become the preferred seed cells for tissue engineering.

After systemic infusion, MSCs can preferentially migrate into sites of inflammation and injury and tumors. This 'homing capacity' of MSCs can be used in drug or gene carrier applications for targeted treatment of diseases or killing of tumor cells [6]. In the situation of renal ischemia-reperfusion injury, MSCs move towards renal tissues and repair ischemic renal tubular injury. Through paracrine and endocrine secretion of various cytokines, MSCs inhibit apoptosis and fibrosis of damaged renal tissues, regulate immune and inflammatory responses, and promote angiogenesis to stimulate renal tissue regeneration instead of directly differentiating into renal tubular epithelial cells [7].

\section{Plasticity of the immunoregulatory and anti-inflammatory properties of MSCs}

MSCs constitutively express low levels of major histocompatibility complex (MHC) class I molecules, but do not express $\mathrm{MHC}$ class II and costimulatory molecules, including B7-1 (CD80), B7-2 (CD86) and CD40 [8], resulting in the absence of a HLA allogeneic barrier during their transplantation. Thus, the lack of immunogenicity of MSCs provides a theoretical basis for their use in extensive transplantation, such that a cord blood bank, for example, could be established.

MSC-mediated immunosuppression is elicited by interferon (IFN) $-\gamma$ in the presence of other proinflammatory cytokines, tumor necrosis factor- $\alpha$, interleukin (IL)$1 \alpha$, or IL-1 $\beta[9,10]$. This activation step has also been shown in vivo in a GVHD model, since recipients of IFN ${ }^{-/-} \mathrm{T}$ cells did not respond to MSC treatment and succumbed to GVHD [11]. The immunosuppression of MSCs was absent in IFN- $\gamma$ receptor $1^{-/-}$mice, highlighting the importance of IFN- $\gamma$ in this process [10].

MSCs exert immunosuppressive effects by secreting bioactive molecules and through cell-to-cell contact (Fig. 1). They can inhibit the activation of macrophages to release proinflammatory cytokines and promote antiinflammatory cytokine secretion. By secreting the soluble antigen IL-6 and prostaglandin $\mathrm{E}_{2}$, MSCs suppress differentiation of monocytes into, and interfere with the development, differentiation and maturation of, dendritic cells. Furthermore, MSCs can reduce the secretion of IFN- $\gamma$ in natural killer cells and change their phenotype, inhibiting their proliferation, cytokine secretion and cell killing role by the activation of Toll-like receptor (TLR)4 [12]. Together with IFN- $\gamma$ stimulation, indoleamine 2,3-dioxygenase (IDO) secreted by MSCs can suppress $\mathrm{T}$-cell proliferation by degrading tryptophan, an essential amino acid for the induction of the T-cell cycle, leading to arrest in G0/G1 phase. MSCs increase CD4 $4^{+}$ $\mathrm{CD}_{25^{+}} \mathrm{FoxP}^{+} \mathrm{T}$ regulatory cell function and can cause the arrest of B lymphocytes in the G0/G1 phase [13]. Mediated by T cells, MSCs inhibit the maturation, migration, proliferation and antibody production of B cells [14].

MSC immunosuppressive functions are 'licensed' by the inflammation environment [10], and this inflammatory status determines the immunoregulatory effect of MSCs. Strong inflammation causes MSCs to suppress the immune response, whereas weak inflammation leads to immune reaction enhancement by MSCs. MSC1 and MSC2 represent the proinflammatory and anti-inflammatory phenotypes of MSCs, respectively [15]. The activation of TLRs can affect the inflammatory functions of MSCs [16]. In the absence of proinflammatory cytokines, the activation of TLR4 can result in the differentiation of MSCs into the MSC1 phenotype. Conversely, differentiation into the MSC2 phenotype can be induced by the delivery of antiinflammatory signals to MSCs through TLR3 $[15,17]$.

\section{MSCs in kidney transplantation}

Since MSCs can exert both anti-ischemia-reperfusion injury and anti-post-transplant rejection functions, these cells are of particular interest in kidney transplantation. Preclinically, MSC therapy positively influences renal function and graft survival $[18,19]$. Since the first successful clinical application of MSCs in 2004 [4], research into MSC therapeutic applications has expanded to the point where there are now more than 500 registered clinical trials (http://clinicaltrials.gov/). However, only nine MSC clinical trials in kidney transplantation have been registered (Table 1). Despite this limited number, translation of MSCs from the bench to bedside for use in kidney transplantation is highly possible.

Reinders et al. [20] have demonstrated that combined treatment with autologous bone marrow-derived MSCs (BM-MSCs) and a maintenance immunosuppressant alleviates acute rejection and interstitial fibrosis/tubular atrophy up to 24 weeks after MSC infusion. Other findings have shown that autologous MSCs injected together with a maintenance immunosuppressant resulted in 


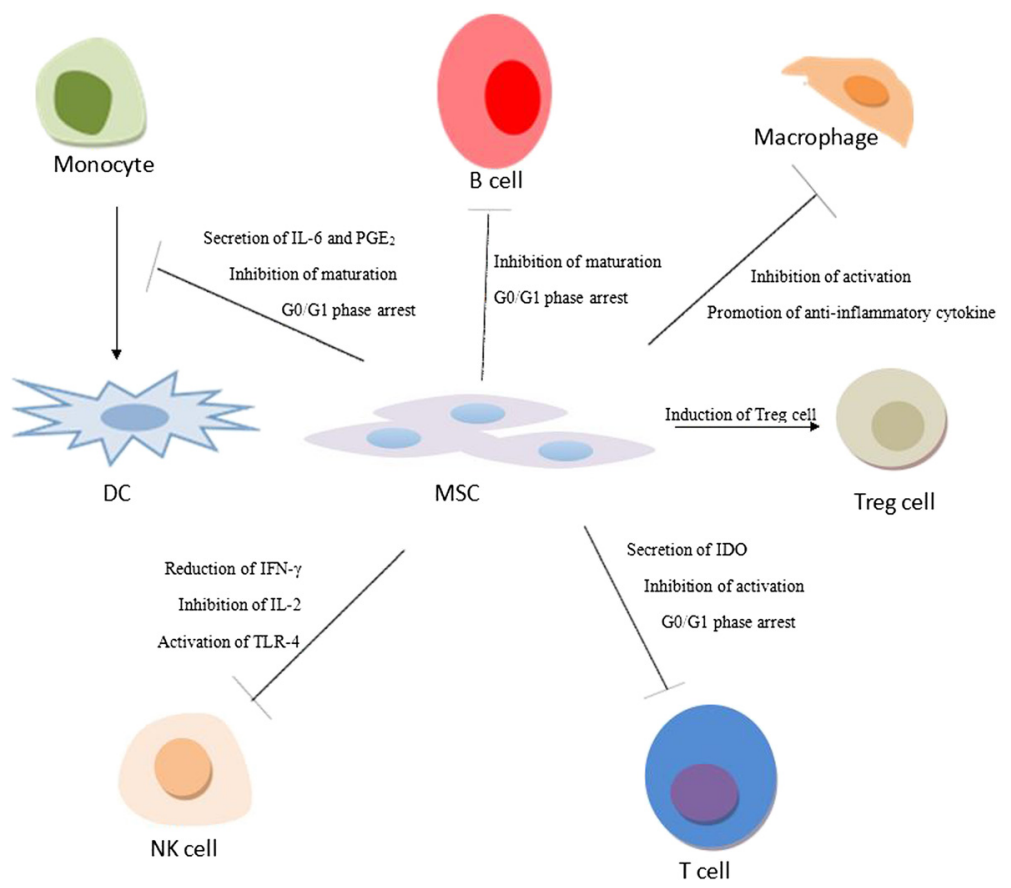

Fig. $1 \mathrm{Imm}$ unomodulatory effects of mesenchymal stem cells (MSCS) on immune cells. MSCs inhibit the activation of macrophages and proliferation of T cells, regulate the activation and differentiation of B cells, and suppress natural killer (NK) cell proliferation. They inhibit monocyte differentiation into dendritic cells $(D C)$ and enhance the cell function of $C D^{+} C d 25^{+}$FoxP $3^{+} T$ regulatory (Treg) cells. They secrete inhibitory factors or make cell-to-cell contacts with various types of immune cells to exert their immunosuppressive effects and treat a variety of immune system disorders. IDO indoleamine 2,3-dioxygenase, IFN interferon, IL interleukin, $P G E_{2}$ prostaglandin $E_{2}$, TLR Toll-like receptor

more stable graft function 1 year after transplantation [21]. Peng et al. [22] described a significant reduction of tacrolimus dose accompanied by stable renal function after allogeneic MSC application. Crop et al. [23] suggested that MSCs can inhibit the proliferation of $\mathrm{T}$ cells and reduce graft inflammation, indicating that MSC administration can be a promising cell therapy in clinical organ transplantation. Vanikar et al. [24] provided evidence that adipose-derived mesenchymal stem cells (ADMSCs) combined with hematopoietic stem cell transplantation perform more effectively than transplantation alone. Results from studies by Trivedi et al.

Table 1 Registered clinical trials of mesenchymal stem cells in kidney transplantation (ClinicalTrial.gov, updated July 2015)

\begin{tabular}{|c|c|c|c|c|c|}
\hline NCT & Status & Title & Site & Type of MSC & Start date \\
\hline NCT02409940 & Recruiting & $\begin{array}{l}\text { To elucidate the effect of mesenchymal stem cells } \\
\text { on the T-cell repertoire of kidney transplant patients }\end{array}$ & Chandigarh, India & $\begin{array}{l}\text { Autologous/allogeneic; } \\
\text { BM-MSC }\end{array}$ & September 2013 \\
\hline NCT02387151 & Recruiting & $\begin{array}{l}\text { Allogeneic mesenchymal stromal cell therapy in } \\
\text { renal transplant recipients }\end{array}$ & Leiden, Netherlands & Allogeneic; BM-MSC & March 2015 \\
\hline NCT02057965 & Recruiting & Mesenchymal stromal cell therapy in renal recipients & Leiden, Netherlands & Autologous; BM-MSC & March 2014 \\
\hline NCT02012153 & Recruiting & $\begin{array}{l}\text { Mesenchymal stromal cells in kidney transplant } \\
\text { recipients }\end{array}$ & Bergamo, Italy & Autologous; BM-MSC & December 2013 \\
\hline NCT00659620 & Unknown & $\begin{array}{l}\text { Mesenchymal stem cell transplantation in the } \\
\text { treatment of chronic allograft nephropathy }\end{array}$ & Fuzhou, Fujian & Autologous; BM-MSC & May 2008 \\
\hline NCT00734396 & Completed & Mesenchymal stem cells and subclinical rejection & Leiden, Netherlands & Autologous; BM-MSC & February 2009 \\
\hline NCT00752479 & Terminated & $\begin{array}{l}\text { Mesenchymal stem cells under basiliximab/low } \\
\text { dose RATG to induce renal transplant tolerance }\end{array}$ & Bergamo, Italy & Autologous; BM-MSC & May 2008 \\
\hline NCT00658073 & Completed & $\begin{array}{l}\text { Induction therapy with autologous mesenchymal } \\
\text { stem cells for kidney allografts }\end{array}$ & Fuzhou, Fujian & Autologous; BM-MSC & March 2008 \\
\hline NCT01429038 & Recruiting & $\begin{array}{l}\text { Mesenchymal stem cells after renal or liver } \\
\text { transplantation }\end{array}$ & Liege, Belgium & Allogeneic; BM-MSC & February 2012 \\
\hline
\end{tabular}


[25] and Vanikar et al. [26] have shown that pretransplant ADMSCs combined with hematopoietic stem cells minimized the overall application of immunosuppressants in kidney transplantation. Tan et al. [27] confirmed that inoculation with MSCs significantly reduced acute rejection incidence and opportunistic infections, while maintaining renal function better than that of controls. Donor MSC injection into the iliac bone at the time of kidney transplantation was reported to be safe [28]. A large-sample meta-analysis has also confirmed the clinical safety of MSC therapies [29].

\section{Problems in the clinical translation of MSCs}

Although MSCs exhibit great potential in most preclinical and clinical data, many questions remain to be resolved. Furthermore, the optimal source of MSCs, the optimal time window, dosage, route and frequency of MSC administration, post-transplantation safety and long-term prognosis have still not been determined.

\section{MSC sources}

MSCs are found in almost all postnatal organs and tissues, including fat, bone, cartilage, umbilical cord, cord blood, synovium, synovial fluid, muscle, skin and pulp. MSCs from different sources possess similar morphologies and express identical surface markers, but they do show differences in some biological characteristics. BM-MSCs are an important part of the bone marrow microenvironment, which promotes the function of hematopoietic stem cells through the secretion of IL-1a, IL-1b, IL-6, IL-7, IL-8, IL11, IL-14, IL-15, leukemia inhibitory factor, stem cell factor, thrombopoietin, fetal liver tyrosine kinase (Flt)-3 ligand, macrophage colony-stimulating factor, granulocyte colony-stimulating factor, and granulocyte-macrophage colony-stimulating factor [30]. Umbilical cord blood mesenchymal stem cells are the youngest MSCs, with the highest potential and lowest immunogenicity [31, 32]. Human umbilical cord MSCs are mainly isolated from Wharton's jelly in the umbilical cord, which can be easily, noninvasively and painlessly obtained without causing adverse effects on the fetus and mother, can be utilized without ethical restrictions, and does not produce teratomas [33]. ADMSCs have many sources, and these cells are easy to isolate and can be repeatedly collected with less pain to the donor. Human amniotic MSCs can be derived from abandoned amniotic membranes and possess low immunogenicity and multi-lineage differentiation, as well as anti-inflammatory abilities similar to those of BM-MSCs. The most promising candidate source of MSCs for cell therapy continues to be debated.

\section{Autologous or allogeneic MSCs}

Thus far, most studies have used autologous cells. Togel et al. [34] reported that autologous MSCs were more potent than allogenic MSCs in a rat acute renal failure model. However, it takes several weeks to months to manufacture autologous cells due to the expansion period, quality controls and logistics, and this period of time is too long for patients in need of treatment. Moreover, MSCs derived from patients with renal disease have a lower capacity for kidney regeneration [35].

Allogeneic MSCs offer an 'off the shelf' advantage for clinical use and have the potential to be mass-produced rapidly [36]. This would significantly decrease costs and the number of procedures, and enable use of cells from young healthy donors that may exhibit higher efficacy than cells from older individuals.

\section{Timing of administration of MSCs}

In a mouse model of GVHD, MSCs were most effective when administered at 3,8 , or 20 days after bone marrow transplantation, and these cells had no protective effect when applied on the day of transplantation, suggesting that a certain proinflammatory environment is necessary for MSCs to polarize into an anti-inflammatory phenotype [11, 17]. In contrast, Casiraghi et al. [37] assumed that better immune suppression by MSCs can be achieved when they are injected before transplantation rather than after it. Perico et al. [21,38] also reported a decrease in kidney function when MSCs were administered within a week after transplantation. Therefore, the optimal timing for MSC administration remains under discussion.

\section{Route of administration of MSCs}

To date, most trials in kidney transplantation have used intravenous infusion of MSCs. However, most of the cells accumulated in the lungs after administration and only a small number of cells reached the transplanted kidney, implying that most MSCs do not play a substantial role in the target tissue. Lung entrapment may be due to the small capillary size, large capillary network, and strong adhesion properties of MSCs. Increasing the number of administered MSCs to compensate for this disadvantage may cause unwanted side effects such as embolism [39]. Zonta et al. [40] reported that local arterial administration is more effective than systemic intravenous administration, while not causing arterial thrombosis, infections, or other adverse reactions.

\section{Dose of MSCs}

The application of MSCs in a dose of $0.4-10 \times 10^{6}$ cells per kilogram of body weight in humans is reportedly the most appropriate, since this dose does not result in significant adverse effects. However, a high-dose $\left(450 \times 10^{6}\right.$ cells) infusion of MSCs in adult sheep was well tolerated and proven safe [41]. 


\section{Frequency of MSC administration}

No long-lasting response was observed in children with steroid-resistant GVHD who received one infusion of MSCs, whereas two or more infusions led to better results [42]. However, Franquesa et al. [43] reported that a single delayed MSC injection was effective for the longterm protection of kidney allografts. Therefore, the most effective number and frequency of MSC infusions are uncertain and still to be determined.

\section{Diversity in the mechanisms of action of MSCs derived from different species}

Murine MSCs exert immunosuppressive effects through nitric oxide generated by inducible nitric oxide synthase, whereas human MSCs use IDO as a major effector molecule. To eliminate species disparities that cause variations in different studies, an IDO-expressing humanized MSC mouse model has been created to mimic the human system [44].

\section{Synergistic role of MSCs with immunosuppressive drugs} Interestingly, MSCs perform better under the conditions of an immunosuppressant-resistant acute inflammatory disease. Le Blanc et al. [4] reported the treatment of severe steroid- and cyclosporine-resistant GVHD with MSCs. Sun et al. [45] described the profound therapeutic effect of human umbilical cord MSCs in severe and refractory systemic lupus erythematosus. Dalal et al. [46] found that patients with severe, non-drug-responsive Crohn's disease can be treated with MSCs. However, the treatment of GVHD with a combination therapy of Prochymal (an MSC-based product) and steroids had no therapeutic effect [47]. The combined administration of MSCs with low-dose cyclosporine A (CsA) reversed the protective influence of CsA and accelerated allograft rejection [48]. Zhang et al. [49] found that, when combined with low-dose CsA, MSCs could not prolong animal survival compared with CsA monotherapy. Therefore, the application of MSCs is most feasible in the therapy of immunosuppressant-resistant patients.

\section{Other potential risks of MSCs}

Other adverse events reported after MSC transplantation include fever, fungal infection, organ system complications (neurological, pulmonary, cardiovascular, gastrointestinal and renal, and hematologic), high recurrence rate, tumor growth and metastasis, and even death [29].

MSCs comprise a rare population of $0.001-0.01 \%$ of all nucleated cells in the bone marrow, so a sufficient number of MSCs cannot be produced in $30 \%$ of patients with recurrent bone marrow biopsy [50]. Moreover, large numbers of MSCs have to be expanded in vitro to obtain amounts needed for clinical treatment and this may change their biological characteristics and result in cell transformation. There is also a risk of microbial contamination during in vitro artificial cell expansion of MSCs. Thus, MSC engraftment requires rigorous bacterial testing, as well as control of the time and dose. Moreover, whether MSCs are traditional 'stem cells' remains controversial. For example, both MSCs and fibroblasts have a similar appearance, surface markers, and differentiation ability. Furthermore, lacking in homogeneity and comparability, current clinical trials on MSC transplantation do not ensure stable and reproducible clinical efficacy. The long-term safety of MSC therapy is also not supported by data from large-scale, clinical, doubleblind, randomized and controlled trials.

\section{Conclusion}

Since its first long-term success in 1954, kidney transplantation has represented the best treatment for patients with ESRD. Immunosuppressive drugs taken by patients after transplantation heighten the risk of opportunistic infections and organ toxicity, which can affect the quality of life of patients as well as graft survival. MSCs have recently emerged as a prominent candidate for cell-based therapies for GVHD [4] and kidney transplantation [22, 27], and in many other clinical areas. Four main characteristics predetermine the clinical applications of MSCs: (1) homing to damaged tissues and inflammatory sites; (2) differentiation into various cell types and tissues; (3) secretion of bioactive molecules; (4) immunomodulation by their immunosuppressive and anti-inflammation properties. The efficacy of MSC therapy depends on the relative amounts of proinflammatory and anti-inflammatory cytokines, and pretreatments with proinflammatory cytokines (such as IFN- $\gamma$ ) improve the efficacy of MSC-based therapy [11]. Therefore, to optimize therapy, feasible approaches and relevant biomarkers are needed so that the inflammatory status of patients at the time of MSC infusion can be monitored.

Numerous preclinical trials have shown that MSCs effectively improve outcome after kidney transplantation, but their value is limited due to the different mechanisms of action of human and murine MSCs. Although many clinical trials have demonstrated promising outcomes of MSC-based therapy, the best source of MSCs, the optimal timing, dosage, route and frequency of MSC administration, as well as long-term post-transplantation safety remain unclear. Nevertheless, we expect that MSCs will contribute substantially to the success of human kidney transplantation, while large-scale, multicenter clinical trials are needed to further validate their clinical effects.

\section{Abbreviations}

ADMSC: Adipose-derived mesenchymal stem cell; BM-MSC: Bone marrowderived mesenchymal stem cell; CSA: Cyclosporine A; ESRD: End-stage renal disease; GVHD: Graft-versus-host disease; HLA: Human leukocyte antigen; 
IDO: Indoleamine 2,3-dioxygenase; IFN: Interferon; IL: Interleukin; MHC: Major histocompatibility complex; MSC: Mesenchymal stem cell; TLR: Toll-like receptor.

\section{Competing interests}

The authors declare that they have no competing interests.

\section{Authors' contributions}

Both authors prepared the outline, wrote the initial draft of the manuscript, edited and revised both the outline and draft of the manuscript, and read and approved the final manuscript.

\section{Published online: 07 February 2016}

\section{References}

1. Hardinger $\mathrm{KL}$, Koch MJ, Brennan DC. Current and future immunosuppressive strategies in renal transplantation. Pharmacotherapy. 2004;24:1159-76.

2. Pittenger MF, Mackay AM, Beck SC, Jaiswal RK, Douglas R, Mosca JD, et al. Multilineage potential of adult human mesenchymal stem cells. Science. 1999;284:143-7.

3. Divya MS, Roshin GE, Divya TS, Rasheed VA, Santhoshkumar TR, Elizabeth KE, et al. Umbilical cord blood-derived mesenchymal stem cells consist of a unique population of progenitors co-expressing mesenchymal stem cell and neuronal markers capable of instantaneous neuronal differentiation. Stem Cell Res Ther. 2012;3:57.

4. Le Blanc K, Rasmusson I, Sundberg B, Gotherstrom C, Hassan M, Uzunel M, et al. Treatment of severe acute graft-versus-host disease with third party haploidentical mesenchymal stem cells. Lancet. 2004;363:1439-41.

5. Dominici M, Le BK, Mueller I, Slaper-Cortenbach I, Marini F, Krause D, et al. Minimal criteria for defining multipotent mesenchymal stromal cells. The International Society for Cellular Therapy position statement. Cytotherapy. 2006;8:315-7.

6. Klopp AH, Gupta A, Spaeth E, Andreeff M, Marini 3rd F. Concise review: Dissecting a discrepancy in the literature: do mesenchymal stem cells support or suppress tumor growth. Stem Cells. 2011;29:11-9.

7. Togel F, Hu Z, Weiss K, Isaac J, Lange C, Westenfelder C. Administered mesenchymal stem cells protect against ischemic acute renal failure through differentiation-independent mechanisms. Am J Physiol Renal Physiol. 2005;289:F31-42.

8. Spaas JH, De Schauwer C, Cornillie P, Meyer E, Van Soom A, Van de Walle GR. Culture and characterisation of equine peripheral blood mesenchymal stromal cells. Vet J. 2013;195:107-13.

9. Krampera M, Cosmi L, Angeli R, Pasini A, Liotta F, Andreini A, et al. Role for interferon-gamma in the immunomodulatory activity of human bone marrow mesenchymal stem cells. Stem Cells. 2006;24:386-98.

10. Ren G, Zhang L, Zhao X, Xu G, Zhang Y, Roberts Al, et al. Mesenchymal stem cell-mediated immunosuppression occurs via concerted action of chemokines and nitric oxide. Cell Stem Cell. 2008;2:141-50.

11. Polchert D, Sobinsky J, Douglas G, Kidd M, Moadsiri A, Reina E, et al. IFNgamma activation of mesenchymal stem cells for treatment and prevention of graft versus host disease. Eur J Immunol. 2008;38:1745-55.

12. Lu Y, Liu J, Liu Y, Qin Y, Luo Q, Wang Q, et al. TLR4 plays a crucial role in MSC-induced inhibition of NK cell function. Biochem Biophys Res Commun. 2015;464:541-7

13. Corcione A, Benvenuto F, Ferretti E, Giunti D, Cappiello V, Cazzanti F, et al. Human mesenchymal stem cells modulate B-cell functions. Blood. 2006;107: 367-72.

14. Rosado MM, Bernardo ME, Scarsella M, Conforti A, Giorda E, Biagini S, et al. Inhibition of B-cell proliferation and antibody production by mesenchymal stromal cells is mediated by T cells. Stem Cells Dev. 2015:24:93-103.

15. Waterman RS, Tomchuck SL, Henkle SL, Betancourt AM. A new mesenchymal stem cell (MSC) paradigm: polarization into a proinflammatory MSC1 or an immunosuppressive MSC2 phenotype. PLoS One. 2010;5, e10088.

16. Raicevic G, Rouas R, Najar M, Stordeur P, Boufker HI, Bron D, et al. Inflammation modifies the pattern and the function of Toll-like receptors expressed by human mesenchymal stromal cells. Hum Immunol. 2010;71: 235-44

17. Bernardo ME, Fibbe WE. Mesenchymal stromal cells: sensors and switchers of inflammation. Cell Stem Cell. 2013;13:392-402.

18. Baulier E, Favreau F, Le CA, Jayle C, Schneider F, Goujon JM, et al. Amniotic fluid-derived mesenchymal stem cells prevent fibrosis and preserve renal function in a preclinical porcine model of kidney transplantation. Stem Cells Transl Med. 2014:3:809-20.

19. De Martino M, Zonta S, Rampino T, Gregorini M, Frassoni F, Piotti G, et al. Mesenchymal stem cells infusion prevents acute cellular rejection in rat kidney transplantation. Transplant Proc. 2010;42:1331-5.

20. Reinders ME, de Fijter JW, Roelofs H, Bajema IM, de Vries DK, Schaapherder $A F$, et al. Autologous bone marrow-derived mesenchymal stromal cells for the treatment of allograft rejection after renal transplantation: results of a phase I study. Stem Cells Transl Med. 2013;2:107-11.

21. Perico N, Casiraghi F, Introna M, Gotti E, Todeschini M, Cavinato RA, et al. Autologous mesenchymal stromal cells and kidney transplantation: a pilot study of safety and clinical feasibility. Clin J Am Soc Nephrol. 2011;6:412-22.

22. Peng $Y$, Ke $M, X u L$, Liu L, Chen $X$, Xia W, et al. Donor-derived mesenchymal stem cells combined with low-dose tacrolimus prevent acute rejection after renal transplantation: a clinical pilot study. Transplantation. 2013;95:161-8.

23. Crop MJ, Baan CC, Korevaar SS, ljzermans JN, Alwayn IP, Weimar W, et al. Donor-derived mesenchymal stem cells suppress alloreactivity of kidney transplant patients. Transplantation. 2009;87:896-906.

24. Vanikar AV, Trivedi HL, Feroze A, Kanodia KV, Dave SD, Shah PR. Effect of co-transplantation of mesenchymal stem cells and hematopoietic stem cells as compared to hematopoietic stem cell transplantation alone in renal transplantation to achieve donor hypo-responsiveness. Int Urol Nephrol. 2011:43:225-32

25. Trivedi HL, Vanikar AV, Kute VB, Patel HV, Gumber MR, Shah PR, et al. The effect of stem cell transplantation on immunosuppression in living donor renal transplantation: a clinical trial. Int J Organ Transplant Med. 2013;4:155-62.

26. Vanikar AV, Trivedi HL, Kumar A, Gopal SC, Patel HV, Gumber MR, et al. Co-infusion of donor adipose tissue-derived mesenchymal and hematopoietic stem cells helps safe minimization of immunosuppression in renal transplantation - single center experience. Ren Fail. 2014;36:1376-84.

27. Tan J, Wu W, Xu X, Liao L, Zheng F, Messinger S, et al. Induction therapy with autologous mesenchymal stem cells in living-related kidney transplants: a randomized controlled trial. JAMA. 2012;307:1169-77.

28. Lee H, Park JB, Lee S, Baek S, Kim H, Kim SJ. Intra-osseous injection of donor mesenchymal stem cell (MSC) into the bone marrow in living donor kidney transplantation: a pilot study. J Transl Med. 2013:11:96.

29. Lalu MM, McIntyre L, Pugliese C, Fergusson D, Winston BW, Marshall JC, et al. Safety of cell therapy with mesenchymal stromal cells (SafeCell): a systematic review and meta-analysis of clinical trials. PLoS One. 2012;7, e47559.

30. Devine SM, Hoffman R. Role of mesenchymal stem cells in hematopoietic stem cell transplantation. Curr Opin Hematol. 2000;7:358-63.

31. Kern S, Eichler H, Stoeve J, Kluter H, Bieback K. Comparative analysis of mesenchymal stem cells from bone marrow, umbilical cord blood, or adipose tissue. Stem Cells. 2006:24:1294-301.

32. Rocha V, Wagner Jr JE, Sobocinski KA, Klein JP, Zhang MJ, Horowitz MM, et al. Graft-versus-host disease in children who have received a cord-blood or bone marrow transplant from an HLA-identical sibling. Eurocord and International Bone Marrow Transplant Registry Working Committee on Alternative Donor and Stem Cell Sources. N Engl J Med. 2000;342:1846-54.

33. Fong CY, Richards M, Manasi N, Biswas A, Bongso A. Comparative growth behaviour and characterization of stem cells from human Wharton's jelly. Reprod Biomed Online. 2007;15:708-18.

34. Togel F, Cohen A, Zhang P, Yang Y, Hu Z, Westenfelder C. Autologous and allogeneic marrow stromal cells are safe and effective for the treatment of acute kidney injury. Stem Cells Dev. 2009;18:475-85.

35. Westerweel PE, Hoefer IE, Blankestijn PJ, de Bree P, Groeneveld D, van Oostrom $\mathrm{O}$, et al. End-stage renal disease causes an imbalance between endothelial and smooth muscle progenitor cells. Am J Physiol Renal Physiol. 2007:292:F1132-40.

36. Trainor N, Pietak A, Smith T. Rethinking clinical delivery of adult stem cell therapies. Nat Biotechnol. 2014;32:729-35.

37. Casiraghi F, Azzollini N, Todeschini M, Cavinato RA, Cassis P, Solini S, et al. Localization of mesenchymal stromal cells dictates their immune or proinflammatory effects in kidney transplantation. Am J Transplant. 2012;12: 2373-83.

38. Perico N, Casiraghi F, Gotti E, Introna M, Todeschini M, Cavinato RA, et al. Mesenchymal stromal cells and kidney transplantation: pretransplant infusion protects from graft dysfunction while fostering immunoregulation. Transpl Int. 2013;26:867-78.

39. Kurtz A. Mesenchymal stem cell delivery routes and fate. Int J Stem Cells. 2008;1:1-7. 
40. Zonta S, De Martino M, Bedino G, Piotti G, Rampino T, Gregorini M, et al. Which is the most suitable and effective route of administration for mesenchymal stem cell-based immunomodulation therapy in experimental kidney transplantation: endovenous or arterial. Transplant Proc. 2010;42: 1336-40.

41. McDonald CA, Oehme D, Pham Y, Kelly K, Itescu S, Gibbon A, et al. Evaluation of the safety and tolerability of a high-dose intravenous infusion of allogeneic mesenchymal precursor cells. Cytotherapy. 2015;17:1178-87.

42. Ball LM, Bernardo ME, Roelofs H, van Tol MJ, Contoli B, Zwaginga JJ, et al. Multiple infusions of mesenchymal stromal cells induce sustained remission in children with steroid-refractory, grade III-IV acute graft-versus-host disease. Br J Haematol. 2013;163:501-9.

43. Franquesa M, Herrero E, Torras J, Ripoll E, Flaquer M, Goma M, et al. Mesenchymal stem cell therapy prevents interstitial fibrosis and tubular atrophy in a rat kidney allograft model. Stem Cells Dev. 2012;21:3125-35.

44. Ling W, Zhang J, Yuan Z, Ren G, Zhang L, Chen X, et al. Mesenchymal stem cells use IDO to regulate immunity in tumor microenvironment. Cancer Res. 2014;74:1576-87.

45. Sun L, Wang D, Liang J, Zhang H, Feng $X$, Wang $H$, et al. Umbilical cord mesenchymal stem cell transplantation in severe and refractory systemic lupus erythematosus. Arthritis Rheum. 2010;62:2467-75.

46. Dalal J, Gandy K, Domen J. Role of mesenchymal stem cell therapy in Crohn's disease. Pediatr Res. 2012;71:445-51.

47. Baker M. Stem-cell drug fails crucial trials. Nat Rep Stem Cells. 2009. doi:10. 1038/stemcells.2009.121.

48. Inoue S, Popp FC, Koehl GE, Piso P, Schlitt HJ, Geissler EK, et al. Immunomodulatory effects of mesenchymal stem cells in a rat organ transplant model. Transplantation. 2006:81:1589-95.

49. Zhang W, Qin C, Zhou ZM. Mesenchymal stem cells modulate immune responses combined with cyclosporine in a rat renal transplantation model. Transplant Proc. 2007:39:3404-8.

50. Yamout B, Hourani R, Salti H, Barada W, El-Hajj T, Al-Kutoubi A, et al. Bone marrow mesenchymal stem cell transplantation in patients with multiple sclerosis: a pilot study. J Neuroimmunol. 2010;227:185-9. 